欧

Artículo de Revisión

\title{
Insuficiencia aórtica severa aguda, aspectos diagnósticos de la ecocardiografía. A propósito de un Caso Clínico
}

\author{
Julián Vega ${ }^{1}$, Samuel Córdova ${ }^{1}$, Luigi Gabrielli ${ }^{1}{ }^{2}$, Paul McNab ${ }^{1}$, María Cecilia Muñoz $^{1}$. \\ 1. División de Enfermedades Cardiovasculares. Escuela de Medicina. \\ Pontificia Universidad Católica de Chile. Santiago, Chile. \\ 2. Advanced Center for Chronic Diseases (ACCDiS), Escuela de Medicina. Pontificia Universidad Católica de Chile. Santiago, Chile. \\ Sin apoyo financiero.
}

La insuficiencia aórtica severa aguda (IASA) constituye una emergencia quirúrgica; sus principales causas son la endocarditis infecciosa y la disección aórtica. Existen tres hallazgos ecocardiográficos distintivos de dicha patología, que ayudan al diagnóstico y manejo que son: el cierre prematuro de la válvula mitral (CPVM), la insuficiencia mitral diastólica (IMD) y la apertura prematura de la válvula aórtica (APVA). Estos elementos reflejan el severo aumento de la presión de fin de diástole del ventrículo izquierdo (PFDVI) y, si bien son específicos, no son únicos de dicha patología. A continuación, se reporta el caso de un paciente con
IASA. Hombre de 36 años, sin antecedentes, consulta por estado infeccioso asociado a insuficiencia cardíaca aguda. Ingresa en shock cardiogénico y la ecocardiografía muestra: ventrículo izquierdo severamente dilatado con función sistólica conservada, dilatación leve de la aurícula izquierda y una endocarditis de válvula aórtica trivalvar asociado a insuficiencia severa. Se identifica, además, la presencia de CPVM y de IMD hallazgos que evidenciaban la severidad de la lesión y lo agudo de la presentación. Se realizó un recambio valvular aórtico de urgencia con una prótesis biológica con buena evolución postoperatoria.

Correspondencia: 


\section{Acute severe aortic regurgitacion echocardiography features: Clinical case and review}

Severe acute aortic regurgitation (SAAR) constitutes a surgical emergency. Its main causes are infective endocarditis and aortic dissection. Three echocardiographic hallmarks aid in its diagnosis and management, namely: premature opening of the aortic valve (POAV), premature mitral valve closure (PMVC) and diastolic mitral regurgitation (DMR), findings that reflect the great increase in left ventricular end-diastolic pressure. Also, these findings are distinctive but not unique to SAAR. We report a 36-year-old male, without past medical history that refers three weeks of malaise, fever and heart failure. At the emergency department, the patient evolved to cardiogenic shock being admitted to the coronary unit. A transthoracic and transesophageal echocardiography revealed a severely dilated left ventricle with normal systolic function, a mild left atrium enlargement and endocarditis of a trileaflet aortic valve with severe regurgitation. Furthermore, PMVC and DMR were identified, findings that portrayed the severe and acute presentation of the disease. A surgical aortic valve replacement was performed uneventfully, and the patient discharged in good conditions.

Key words: Aortic valve regurgitation, Heart failure, Endocarditis, Echocardiography 


\section{Introducción:}

La insuficiencia aórtica severa cuando se presenta de forma aguda (IASA) constituye una emergencia que habitualmente requiere pronta resolución quirúrgica. Sus principales causas son la endocarditis infecciosa y la disección aórtica ${ }^{1}$. La ecocardiografía es clave para su diagnóstico y manejo, permitiendo reconocer elementos distintivos de la IASA. Asimismo, otorga información sobre morfología y función ventricular, otras alteraciones cardíacas asociadas y características de la aorta torácica, guiando la reanimación y manejo del paciente. A continuación, exponemos un caso de IASA haciendo énfasis en los aspectos diagnósticos de la ecocardiografía.

\section{Caso clínico:}

Hombre de 36 años sin antecedentes médicos, acude a urgencias por estado infeccioso asociado a disminución de su capacidad funcional de tres semanas de evolución. El examen físico constata un paciente en malas condiciones generales, mal perfundido, taquicárdico, hipotenso y un soplo de insuficiencia aórtica. En la unidad corona-
Figura 2. Cierre prematuro de la válvula mitral (CPVM).

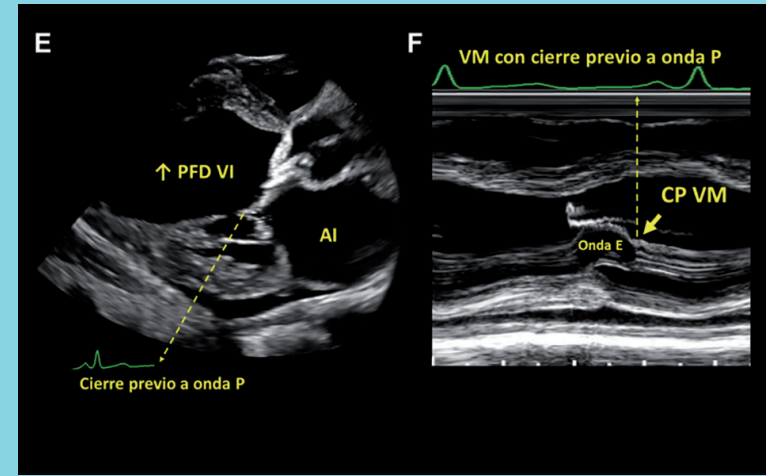

Panel E. ETT: Eje largo paraesternal, se evidencia cierre valvular mitral previo a la onda P del ECG.

Panel $\boldsymbol{F}$. ETT con modo $M$ de la válvula mitral, se observa la completa relación temporal del cierre valvular mitral previo a la onda P del ECG y la consiguiente ausencia de onda A mitral.

Leyenda: AI: Aurícula izquierda. PFD VI: Presión de fin de diástole del ventrículo izquierdo. VM: Válvula mitral. CP VM: Cierre prematuro de la válvula mitral.

Material complementario disponible en http://www.ecocardio.clliasa.html

ria se realiza una ecocardiografía transtorácica (ETT) y transesofágica (ETE) (PHILIPS EPIC 7c, Andover, MA,

Figura 1. Imágenes ecocardiográficas del caso reportado.

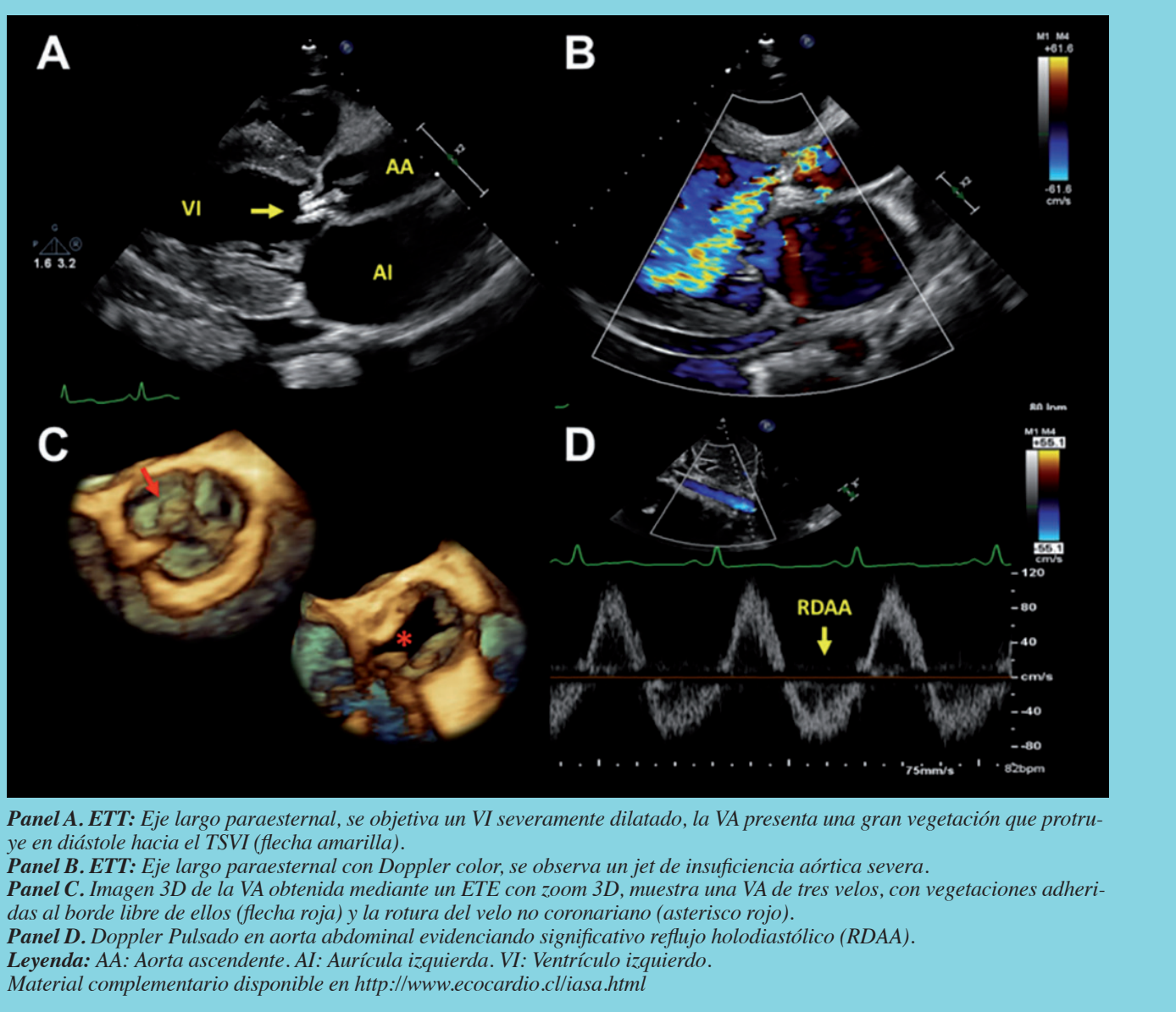




\section{Figura 3. Insuficiencia mitral diastólica (IMD).}

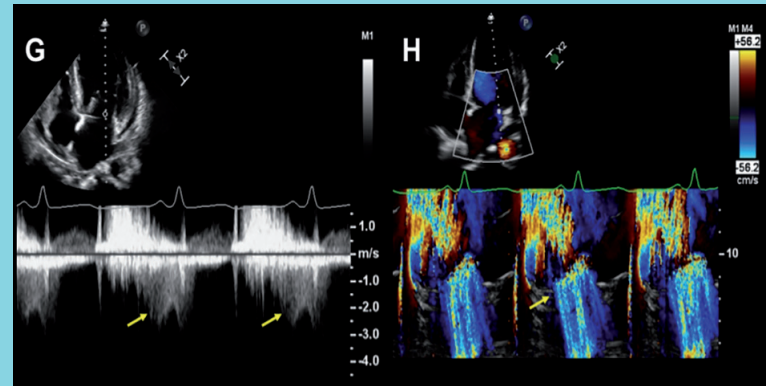

Panel G. ETT vista apical de 4 cámaras con Doppler continuo sobre la válvula mitral, se aprecia la presencia de insuficiencia mitral diastólica (flechas amarillas).

Panel H. ETT vista apical de 4 cámaras con modo M color sobre la válvula mitral, se aprecia la relación temporal entre la insuficiencia mitral (flecha amarilla) y la diástole del ECG.

Material complementario disponible en

http://www.ecocardio.cl/iasa.html

USA), que describen: ventrículo izquierdo (VI) severamente dilatado con un volumen 3D de fin de diástole indexado por superficie corporal de $128 \mathrm{ml} / \mathrm{m} 2$. La motilidad global y segmentaria se encontraba conservada (FEVI 55\% por Simpson biplano) y la aurícula izquierda se encontraba levemente dilatada $(38 \mathrm{ml} / \mathrm{m} 2$ de volumen biplano indexado). La válvula aórtica (VA) presentaba tres velos con múltiples vegetaciones protruyendo hacia el tracto de salida del ventrículo izquierdo (Figura 1, Panel A), identificando una perforación en la base del velo coronariano derecho (Figura 1, Panel B y C), asociado a rotura del velo no coronariano generando insuficiencia aórtica severa. Además, en la aorta abdominal se registró reflujo holodiastólico (Figura 1, Panel D). Sumado a lo anterior la válvula mitral presentaba cierre prematuro, el que ocurría previo a la onda P del ECG (Figura 2, Panel E), causando ausencia de la onda A en el Doppler pulsado del flujo transmitral. Este fenómeno fue mejor evidenciado en el modo $\mathrm{M}$ debido a su alta resolución temporal (Figura 2, Panel F). Otro hallazgo registrado fue la pre- sencia de insuficiencia mitral diastólica (Figura 3). Estos elementos denotan la severidad y lo agudo de la presentación clínica. El deterioro de la condición hemodinámica justificó cirugía cardíaca de urgencia. Los hallazgos del intraoperatorio confirmaron una válvula aórtica de tres velos con vegetaciones en todos ellos, identificando rotura del velo coronariano derecho y del velo no coronariano. Se realizó un reemplazo valvular aórtico con prótesis biológica Carpentier Edwards Perimount de $25 \mathrm{~mm}$ sin incidentes. Los hemocultivos resultaron positivos para Streptococcus Gallolyticus ajustando la terapia antibiótica. La evolución en el postoperatorio fue favorable recibiendo el alta luego de 15 días de estadía hospitalaria.

\section{Discusión:}

Las principales causas y mecanismos de la IASA se resumen en la Tabla 1, la etiología más frecuente es la endocarditis infecciosa, que puede presentarse en una válvula bicúspide (VAB) o tricúspide. Cuando la VA presenta previamente insuficiencia, como se presume que ocurrió en el caso reportado debido al hallazgo de una VAB, dicha situación permite al ventrículo izquierdo remodelarse y tolerar parcialmente la aparición súbita de insuficiencia aórtica severa, la que generalmente es causada por una perforación valvular en el contexto de una endocarditis. Menos frecuente es la aparición de insuficiencia aórtica severa aguda sobre una válvula sin insuficiencia previa, escenario mal tolerado y que se presenta en shock cardiogénico. No obstante las diferencias en su presentación clínica, ambas situaciones deben ser resueltas quirúrgicamente de forma prioritaria. Y la premura quirúrgica depende de la tolerancia hemodinámica del paciente, donde la ecocardiografía aporta información decisiva. Debido a esto, es útil comprender los hallazgos ecocardiográficos asociados a IASA, y que se describen a continuación, junto con un breve resumen de su fisiopatología.

Tabla 1. Principales causas y mecanismos de la IASA.

\begin{tabular}{|c|c|c|}
\hline Etología & Frecuencia & Mecanismos \\
\hline Endocarditis infecciosa & $55 \%$ & - Destrucción, perforación valvular o absceso perivalvular roto \\
\hline Disección aórtica & $44 \%$ & $\begin{array}{l}\text { - Dilatación del anillo con falta de coaptación de los velos } \\
\text { - Compromiso comisural con pérdida de soporte de los velos } \\
\text { - Extensión de la disección hacia la base de los velos } \\
\text { - Prolapso del flap de disección hacia el plano valvular }\end{array}$ \\
\hline Disfunción protésica & \multirow{4}{*}{$<1 \%$} & $\begin{array}{l}\text { - Disfunción de una prótesis valvular, prótesis mecánica bivalva con atascamiento de } \\
\text { uno de sus hemidiscos }\end{array}$ \\
\hline $\begin{array}{l}\text { Rotura espontánea de } \\
\text { una fenestración aórtica }\end{array}$ & & $\begin{array}{l}\text { - Rotura espontánea de una fenestración de un velo a lo largo de su línea de cierre } \\
\text { - Rotura espontánea del Rafe una válvula bicúspide }\end{array}$ \\
\hline Trauma torácico & & - Contusión cardíaca con rotura valvular \\
\hline latrogénica & & $\begin{array}{l}\text { - Rotura valvular en contexto de intervencionismo cardíaco: posterior balón plastia, } \\
\text { TAVI o intervencionismo coronario. }\end{array}$ \\
\hline
\end{tabular}




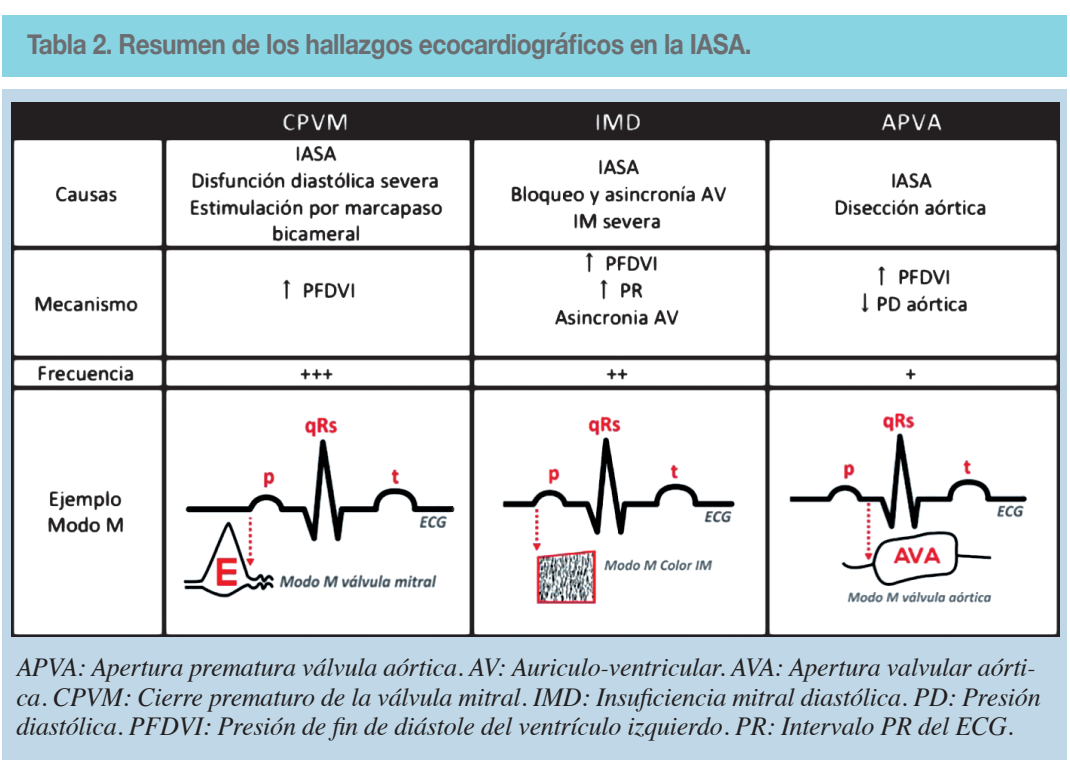

\section{Fisiopatología}

En la IASA existe una súbita sobrecarga de volumen en un ventrículo izquierdo poco preparado, generando una súbita y severa elevación de la PFDVI ( $>30 \mathrm{mmHg}$ ), la que puede igualar la presión diastólica aórtica y superar la presión diastólica de la aurícula izquierda. Lo anterior incrementa la presión capilar pulmonar, disminuye el volumen expulsivo y genera caída de la presión de perfusión coronaria, existiendo como compensación vasoconstricción periférica y taquicardia para mantener el gasto cardíaco.

\section{Aspectos diagnósticos de la ecocardiografía}

Además de los hallazgos ecocardiográficos clásicos que definen severidad en insuficiencia aórtica, en la IASA existen tres hallazgos específicos, pero no exclusivos, que están determinados por la presentación aguda de la insuficiencia y que se describen a continuación (Tabla 2).

\section{Cierre prematuro de la válvula mitral (CPVM)}

Se define como el cierre valvular mitral que ocurre antes de la onda Q del ECG. Pridie en $1971^{2}$ fue el primer autor en describir dicho fenómeno mediante ecocardiografía con modo $\mathrm{M}$, que debido a su alta resolución temporal es el mejor método para detectarlo. Desde entonces este hallazgo ha sido reportado en un $30 \%$ de las IASA. Su causa es la elevada PFDVI que distiende rápidamente un ventrículo izquierdo no remodelado, traccionando al aparato subvalvular y generando por gradiente de presión el cierre mitral anticipado, lo normal es que ocurra $40 \mathrm{~ms}$ luego del complejo QRS. Este fenómeno protege, inicial y parcialmente, a la circulación pulmonar de la transmisión retrógrada de las elevadas PFDVI. El CPVM puede ocurrir con distintas temporalidades lo que tiene implicancia pronóstica ${ }^{3}$ :

- CPVM precoz (Tipo I): Se presenta en proto-meso-diástole, precede a la onda P del ECG y ocurre posterior a la onda $E$ del flujo transmitral. En este escenario la contracción auricular no logra superar la presión ventricular generando ausencia de onda A en el llenado trans-

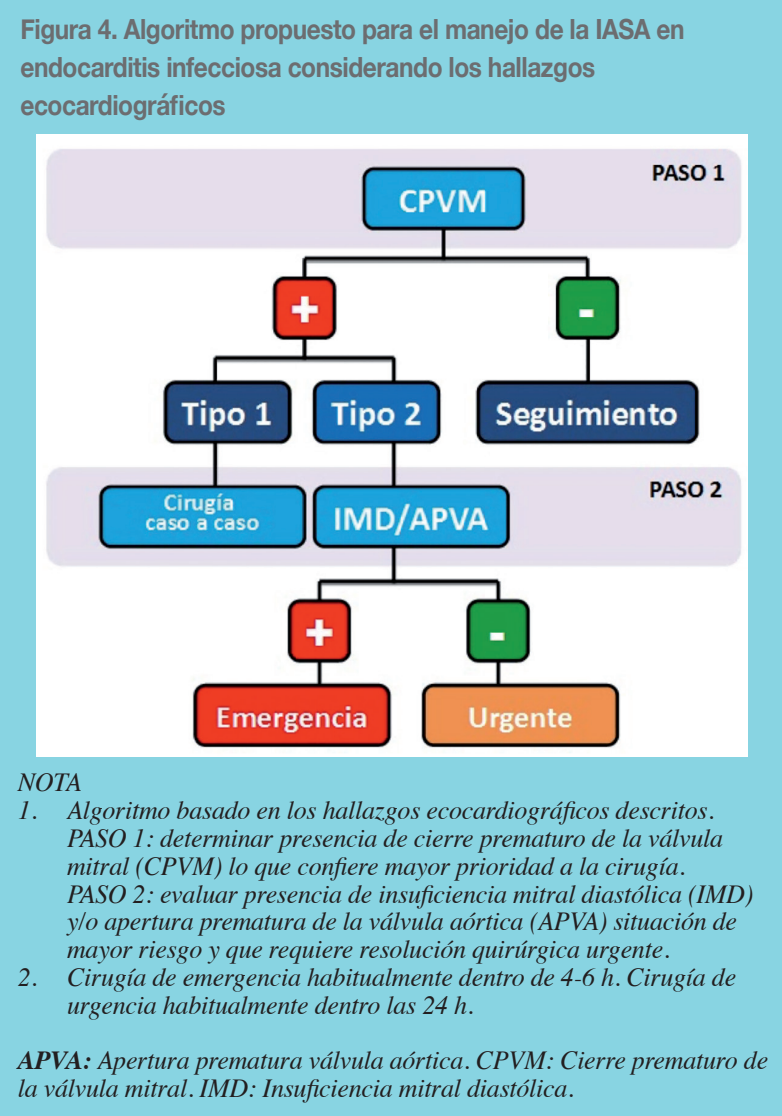


mitral. Es un signo de mal pronóstico, que traduce mayor PFDVI y, por tanto, de peor tolerancia hemodinámica.

- CPVM más tardío (Tipo II): Se presenta en tele-diástole, cercano a la onda Q del ECG y genera en el Doppler del flujo transmitral una onda A "truncada", escenario mejor tolerado, ya que existe un mayor llenado ventricular y mayor volumen expulsivo latido.

\section{Insuficiencia mitral diastólica (IMD)}

Hallazgo que se presenta en magnitud variable hasta en la mitad de los pacientes con IASA. Como se comentó, el CPVM en una fase inicial protege parcialmente la transmisión de forma retrógrada de las elevadas PFDVI. Sin embargo, el progresivo incremento de la PFDVI genera la re-apertura de la válvula mitral hacia el fin de la diástole generando IMD, deteriorando aún más la condición hemodinámica. Este hallazgo, sin embargo, no es específico de la IASA ya que puede observarse en trastornos de la conducción auriculo-ventricular (bloqueo AV de cualquier grado), fibrilación y flutter auricular, insuficiencia mitral sistólica severa y disfunción diastólica severa (fisiología restrictiva).

3. Apertura prematura (diastólica) de la válvula aórtica (APVA). Fenómeno excepcional reportado originalmente por Freizi y cols en $1974^{4}$. Para su aparición requiere de una elevación severa de la PFDVI, que iguale y supere brevemente la presión diastólica aórtica, la que suele encontrarse disminuida. Lo anterior genera apertura valvular aórtica (VA) prematura hacia el final de la diástole, fenómeno que se hace más evidente a frecuencias cardíacas bajas. Reportes iniciales mencionaban como posible mecanismo en presencia de un equilibrio de las presiones diastólicas entre aorta y VI, que la válvula aórtica podría abrirse de forma pasiva ("flotar abierta" al existir un mínimo gradiente entre el VI y la aorta). Sin embargo, observaciones posteriores sugieren que la APVA se da como respuesta a la sístole auricular sumado un efecto elástico ("recoil") del ventrículo izquierdo que se transmite hacia la VA.

Cabe destacar que este último hallazgo requiere la presencia conjunta de una PFDVI severamente elevada y de una presión diastólica aórtica disminuida, situación más factible en el escenario de una insuficiencia aórtica crónica en rango moderado que progresa súbitamente a severa. Es importante señalar que este hallazgo tampoco es específico de IASA, pues de forma excepcional ha sido reportado en ausencia de insuficiencia aórtica, en casos de disección aórtica, con compresión del falso lumen, causando disminución de la presión diastólica aórtica, sin asociarse a elevación de la PFDVI.

\section{Otros hallazgos}

Existen otros elementos que orientan a que la insuficiencia aórtica es un fenómeno agudo, como son un ventrículo izquierdo poco remodelado (dilatado), con aspecto hiperdinámico tipo sobrecarga de volumen y la presencia de hipertensión pulmonar significativa.

\section{Conclusiones:}

Los hallazgos mencionados aportan información trascendental sobre la tolerancia hemodinámica del paciente a la IASA y colaboran en determinar la urgencia quirúrgica. Así por ejemplo; un paciente que presenta CPVM de forma muy precoz (preceda la onda P del ECG) asociado a insuficiencia mitral diastólica se encuentra en una situación crítica que requiere cirugía de emergencia, en contraste a un paciente que presenta CPVM tardío y sin insuficiencia mitral diastólica asociada, en la figura 4 se propone un algoritmo de manejo basado en los hallazgos ecocardiográficos descritos. Es relevante recalcar que los signos ecocardiográficos descritos CPVM, IMD y ADVA son marcadores de aumento severo de la PFDVI y, por tanto, no son $100 \%$ específicos para IASA, ya que pueden darse excepcionalmente en ausencia de insuficiencia aórtica en las situaciones antes señaladas, recomendando siempre su interpretación asociado al contexto clínico del paciente.

\section{Referencias}

1. HAMIRANI YS, DIETL CA, VOYLES W, PERALTA M, BEGAY D, RAIZADA V. Acute aortic regurgitation. Circulation. 2012;126:1121-1126.

2. PRIDIE RB, BENHAM R, OAKLEY CM. Echocardiography of the mitral valve in aortic valve disease. Br Heart J. 1971;33:296-304
3. BOTVINICK EH, SCHILLER NB, WICKRAMASEKARAN R, KLAUSNER SC, GERTZ E. Echocardiographic demonstration of early mitral valve closure in severe aortic insufficiency. Its clinical implications. Circulation. 1975;51:836-847.

4. FEIZI O, SYMONS C, YACOUB M. Echocardiography of the aortic valve. I. Studies of normal aortic valve, aortic stenosis, aortic regurgitation, and mixed aortic valve disease. Br Heart J. $1974 ; 36: 341-351$ 